\title{
Distribución y abundancia del pez león Pterois volitans (Scorpaeniformes: Scorpaenidae) y especies nativas asociadas en el Parque Marino Cayos de San Felipe, Cuba
}

\author{
Elena de la Guardia ${ }^{1 *}$, Dorka Cobián Rojas ${ }^{2}$, Leonardo Espinosa ${ }^{3}$, Zaimiuri Hernández $^{3}$, \\ Lázaro García ${ }^{3} \&$ Jesús Ernesto Arias González ${ }^{1 *}$ \\ 1. Centro de Investigación y de Estudios Avanzados del I. P. N. - Mérida, Dep. de Recursos del Mar, Yucatán, Ant. Carr. \\ a Progreso Km. 6, A.P. 73 Cordemex, 97310, México; elenadelaguardia@yahoo.com, earias@cinvestav.mx \\ 2. Parque Nacional Península Guanahacabibes, Centro de Investigaciones y Servicios Ambientales, La Bajada, 22100, \\ Pinar del Río, Cuba; dorkacobianrojas79@gmail.com \\ 3. Parque Nacional Cayos de San Felipe, La Coloma, 20100, Pinar del Río, Cuba; florafpr@enet.cu \\ 4. Centro de Investigaciones Marinas, Universidad de La Habana, Calle 16 No. 114, Paya, Habana, CP 11300, Cuba; \\ lg1@cim.uh.cu \\ * Correspondence
}

Recibido 16-IX-2015. C Corregido 04-VIII-2016. Aceptado 06-IX-2016.

\begin{abstract}
Distribution and abundance of the lionfish Pterois volitans (Scorpaeniformes: Scorpaenidae) and associated native species in Parque Marino Cayos de San Felipe, Cuba. The first lionfish sighting at the National Park "Cayos de San Felipe" was in 2009 and could be a threat to its marine ecosystem diversity and their capacity to generate services. To analyze the incidence of the lionfish invasion in the area, an annual sampling was conducted between 2013 and 2015. Lionfish abundance and size was investigated on mangroves through visual census on ten transects of $30 \times 2 \mathrm{~m} /$ station, and on coral reefs ( 15 and $25 \mathrm{~m}$ deep) with stereo video on six transects of 50x2 m/station. Additionally, incidence of potential native competitors and predators on coral reefs were also estimated. Over the three years, the average density of lionfish varied between $0.0-1.3$ indiv./100 $\mathrm{m}^{2}$ per sample stations and it was not significantly different among habitats (mangroves with 0.6 indiv./100 $\mathrm{m}^{2}$, reefs at $15 \mathrm{~m}-0.4$ indiv. $/ 100 \mathrm{~m}^{2}$ and reef at $25 \mathrm{~m}$ with 0.3 indiv. $/ 100 \mathrm{~m}^{2}$ ). Lionfish's density was equal to or lower than competitors' density, and was equal to or higher than predator's density in both depths. While lionfish density on mangroves and on reefs at $25 \mathrm{~m}$ remained temporally stable, it decreased on reefs at $15 \mathrm{~m}$. Temporary increase in the competitor's density was observed and the predator's density did not change during the monitored time. Lionfish size varied between 5 and $39 \mathrm{~cm}$; the average fish size from mangroves $(12.6 \mathrm{~cm})$ was consistently lower than from reefs $(25.2 \mathrm{~cm})$ and showed no variations among years. Lionfish size in reefs was higher than competitor's size and lower than that of predator. Results showed that in the park: 1) mangroves represent lionfish nursery areas; 2) incidence of reef lionfish was not as high as in other areas of Cuba and the Caribbean; and 3) lionfish abundance in reefs tended to decrease over the years, without the intervention of extractive activities or high abundance of large size native groupers. In this sense, recommendations are made to continue monitoring and to investigate lionfish effects and factors that are regulating its incidence in the park. Rev. Biol. Trop. 65 (1): 117-125. Epub 2017 March 01.
\end{abstract}

Key words: lionfish, invasive species, coral reef, Caribbean, size, density.

El establecimiento del pez león (Pterois volitans: Scorpaenidae) en la región del Gran Caribe ha sido considerado de forma general como una amenaza potencial para la biodiversidad de los ecosistemas marinos (Betancur et al., 2011; Valdez-Moreno, Quintal-Lizama,
Gómez-Lozano, \& García-Rivas, 2012). Se plantea que su presencia puede llegar a afectar la conducta, la distribución, el crecimiento y el tamaño de poblaciones de peces o invertebrados nativos, llegando a reducir la diversidad de especies o modificar tramas tróficas (Albins, 
2013; Bejarano, Lohr, Hamilton, \& Manfrino, 2015). Incluso, estudios hechos a partir de modelos plantean que la presencia del pez león puede llegar a deteriorar la capacidad del ecosistema para brindar servicios (Arias-González, González-Gandara, Cabrera, \& Christensen, 2011). No obstante, el éxito en su colonización, y por tanto el impacto que el pez león pueda llegar a tener, está influenciado por numerosos factores naturales y humanos (Côté \& Green, 2012; Valdivia, Bruno, Cox, Hackerott, \& Green, 2014).

El primer registro del pez león dentro del Parque Nacional Cayos de San Felipe (PNCSF) fue realizado por pescadores en agosto de 2009 $\mathrm{y}$, aunque no se han realizado publicaciones sobre su avistamiento o incidencia en el área, se supone que como en otras zonas de Cuba (Chevalier et al., 2014; García-Rodríguez, Chevalier, Cabrera, Caballero, \& Hernández, 2015) el pez león haya tenido una colonización exitosa en el PNCSF. Este criterio se basa en que los pastos marinos, manglares y arrecifes que caracterizan el parque, están en buen estado de salud y brindan un ambiente favorable para la alimentación, el refugio y la reproducción del pez león. También, porque el pez león no es objeto de la pesca de peces multi-especie que tiene lugar en los Cayos de San Felipe y la zona adyacente (Claro, Baisre, Lindeman, \& García-Arteaga, 2001), y sin embargo, este tipo de pesca si pudiera incidir de forma negativa en la abundancia de especies de meros nativas con potencialidades de regular la presencia del pez león por competencia o depredación (Betancur-R et al., 2011; Mumby, Harborne, \& Brumbaugh, 2011), y con ello, dar ventajas al pez león en la colonización de los hábitats marinos del PNCSF.

Esta investigación analizó la incidencia de Pterois volitans y de algunas especies nativas posibles competidores y depredadores del pez león en manglares y arrecifes del PNCSF entre 2013 y 2015. El objetivo fue caracterizar el comportamiento temporal y espacial de la densidad y la talla de estas especies, con el propósito de obtener una línea base y proponer estrategias para su investigación, monitoreo y manejo.

\section{MATERIALES Y MÉTODOS}

Área de estudio: El PNCSF se localiza en el Golfo de Batabanó (Fig. 1) y dentro de sus límites están incluidos $242 \mathrm{~km}^{2}$ de área marina, con extensos manglares (principalmente

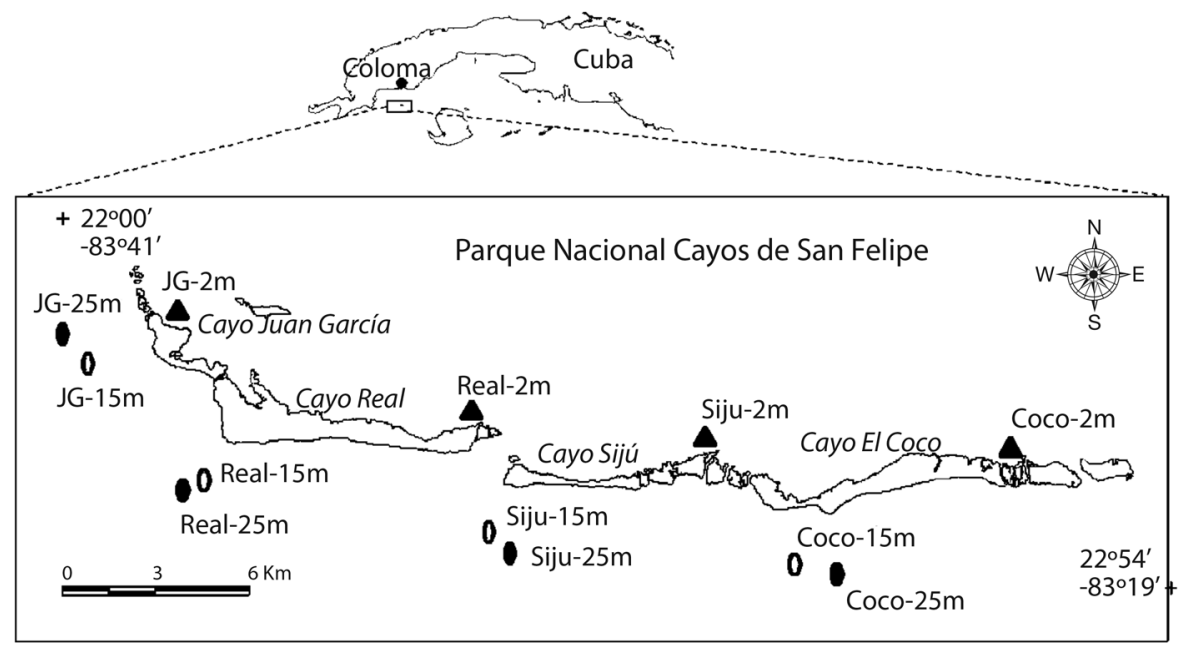

Fig. 1. PNCSF y estaciones de muestreo. Estaciones en mangle a $2 \mathrm{~m}$ de profundidad (triángulos), estaciones en arrecife a $15 \mathrm{~m}$ (círculos sólidos) y estaciones en arrecife a $25 \mathrm{~m}$. (círculos abiertos).

Fig. 1. Location of the PNCSF and sample stations. Mangroves stations at $2 \mathrm{~m}$ deep (triangles), reef stations at $15 \mathrm{~m}$ (solid circle), reef stations at $25 \mathrm{~m}$ (open circle). 
Rhizophora mangle), pastos marinos y cerca de $40 \mathrm{~km}$ de arrecifes de coral al sur de los cayos. En los arrecifes predominan las cordilleras entre 12 y $30 \mathrm{~m}$ de profundidad, con alta representatividad de corales de los géneros Orbicella, Porites y Siderastrea.

Muestreo: Se efectuó un muestreo anual entre 2013 y 2015 en cuatro sitios ubicados cerca de los cayos Juan García (JG), Real, Sijú y Coco (cayos principales del PNCSF). En cada sitio se ubicó una estación en mangle (1-2 $\mathrm{m}$ de profundidad) y dos en arrecife (15 y $25 \mathrm{~m}$ de profundidad), para un total de cuatro estaciones en mangle y ocho en arrecife (Fig. 1). Para minimizar la variabilidad estacional, todos los muestreos se realizaron entre octubre y noviembre en horarios comprendidos entre las 10-16 horas.

Las estaciones en mangle se ubicaron en zonas de canales con entramado complejo y en ellas se cuantificó la presencia de pez león en 10 transectos lineales de banda (30x2 m) con la técnica de censo visual. Para estimar la talla de los individuos observados se utilizaron las siguientes clases: $<5 \mathrm{~cm} ; 5-10 \mathrm{~cm} ; 10-20 \mathrm{~cm}$; 20-30 cm; 30-40 cm; $>40 \mathrm{~cm}$.

Las estaciones de muestreo en arrecife se ubicaron en zonas con heterogeneidad del paisaje y rugosidad del sustrato similar y en ellas se cuantificó la presencia del pez león y de sus posibles competidores y depredadores en seis videos de transectos lineales de banda (50x2 m) separados por intervalos de $10 \mathrm{~m}$. El operador de las cámaras nadó en línea recta a lo largo del arrecife a velocidad constante $(\approx$ 3-4 min por transecto) y a una altura de $50 \mathrm{~cm}$ por arriba del fondo con las cámaras apuntando suavemente hacia el fondo $\left(\approx 30^{\circ}\right.$ con relación al fondo). Como posibles competidores se consideraron Cephalopholis fulva, C. cruentata, Epinephelus guttatus y E. adscensionis, especies de meros de talla mediana que ocupan hábitats similares $\mathrm{y} / \mathrm{o}$ consumen presas parecidas a las reportadas para el pez león (Morris \& Akins, 2009). Como posibles depredadores se seleccionaron Mycteroperca spp. y E. striatus, especies de meros de talla grande ( $>30 \mathrm{~cm} \mathrm{LT})$ identificadas como potenciales consumidoras del pez león (Mumby et al., 2011). Las imágenes tomadas con la técnica de estéreo video se analizaron posteriormente con el programa EventMeasure versión 3.32 (SeaGIS, 2011) para contar y medir individuos en estaciones de arrecife. Con esta técnica se han obtenido resultados precisos y comparables a los de censo visual en la estimación de abundancia y talla de peces de arrecifes (Harvey, Fletcher, Shortis, \& Kendrick, 2004).

Análisis de datos: Los valores de abundancia se estandarizaron mediante su expresión en densidad (indiv. $/ 100 \mathrm{~m}^{2}$ ). Para conocer la variabilidad en la densidad y la talla del pez león y de los grupos de meros, posibles competidores y depredadores en el PNCSF, durante el período de muestreo, los datos se agruparon por estaciones y hábitats (mangle $2 \mathrm{~m}$, arrecife 15 m y $25 \mathrm{~m}$ ) para cada año por separado. Como medida de dispersión de los valores se utilizaron estimadores asociados a los intervalos de confianza del $95 \%$ (límite inferior y límite superior). La significación estadística de las diferencias observadas en los valores medios de densidad y talla de cada uno de los grupos de especies estudiadas se verificó con análisis de varianza (ANOVA) bifactorial, donde los factores fueron hábitat y años.

Para caracterizar el comportamiento de la densidad y la talla promedio del pez león con relación a la de los meros, posibles competidores y depredadores, los datos se agruparon por hábitats (los tres años juntos) y por años para el arrecife (15 y $25 \mathrm{~m}$ de profundidad juntos). La significación estadística de las diferencias observadas en los valores medios, entre hábitats y entre años, se verificó con ANOVA de una vía. La normalidad y homogeneidad de varianza de los datos se comprobó siguiendo los criterios de Underwood (1997) y, en los casos en que los ANOVAs dieron diferencias significativas se aplicó la prueba de comparación de medias de Student-Newman-Keuls (SNK). Los cálculos se ejecutaron con el programa STATISTICA 8.0. 


\section{RESULTADOS}

Abundancia y la talla de pez león y grupos de especies depredadoras o competidoras: Se contaron 91 peces león y se estimó la talla de 147 individuos (Cuadro 1). En el PNCSF, la densidad promedio del pez león por estación varió entre 0 y 1.3 indiv. $/ 100 \mathrm{~m}^{2}$ y su talla osciló entre 8.3 y $30.4 \mathrm{~cm}$. En manglares la densidad promedio fue de $0.65,0-5$ indiv./100 $\mathrm{m}^{2}$ (promedio, mínimo-máximo) y su talla de 12.6, 5-25 cm; en arrecife a $15 \mathrm{~m}$ la densidad promedio fue de $0.41,0-3$ indiv. $/ 100$ $\mathrm{m}^{2}$ y la talla fue de $24.1,10-36 \mathrm{~cm}$; y a $25 \mathrm{~m}$ se encontró densidad promedio de $0.32,0-3$ indiv. $/ 100 \mathrm{~m}^{2} \mathrm{y}$ talla de $25.8,15-39 \mathrm{~cm}$ (Fig. 2A y Fig. 2B). La densidad promedio de pez león fue similar en los tres hábitats $\left(\mathrm{F}_{(2,235)}=2.57 \mathrm{P}=\right.$ $0.08) \mathrm{y}$, aunque en arrecifes a $15 \mathrm{~m}$ fue menor en $2015\left(\mathrm{~F}_{(2,69)}=5.32 \mathrm{P}=0.007\right)$ y tendió a disminuir durante el período de muestreo, no se encontraron diferencias estadísticas entre años $\left(\mathrm{F}_{(2,235)}=2.64 \mathrm{P}=0.07\right)$. La talla promedio fue menor en manglares $\left(\mathrm{F}_{(2,143)}=61.3 \mathrm{P}=0.00\right) \mathrm{y}$ similar entre años $\left(\mathrm{F}_{(2,143)}=1.89 \mathrm{P}=0.15\right)$.

Se contaron 112 posibles competidores y se midieron 179 ejemplares, las especies de meros posibles competidoras con el pez león más comunes en los arrecifes fueron C. fulva, E. guttatus, E. adscensionis y C. cruentata

CUADRO 1

Número de pez león y meros potenciales competidores o depredadores avistados en las estaciones de muestreo

TABLE 1

Number of lionfish and groupers potential competitors or predators sightings by sampling stations

\begin{tabular}{|c|c|c|c|c|c|c|}
\hline \multicolumn{2}{|c|}{ Pez león } & JG & Real & Siju & Coco & Total \\
\hline \multirow[t]{3}{*}{ Mangle $\cdot 2 \mathrm{~m}$} & 2013 & $2 / 4$ & $5 / 7$ & $2 / 5$ & $8 / 8$ & $17 / 24$ \\
\hline & 2014 & ND & $2 / 2$ & $3 / 3$ & $8 / 8$ & $13 / 13$ \\
\hline & 2015 & $4 / 6$ & $2 / 5$ & ND & $3 / 4$ & $9 / 15$ \\
\hline \multirow[t]{3}{*}{ Arrecife $\cdot 15 \mathrm{~m}$} & 2013 & $8 / 8$ & $5 / 5$ & $5 / 5$ & $0 / 2$ & $18 / 20$ \\
\hline & 2014 & $1 / 2$ & $3 / 3$ & $3 / 3$ & $2 / 4$ & $9 / 12$ \\
\hline & 2015 & $0 / 6$ & $1 / 2$ & $2 / 5$ & $0 / 4$ & $3 / 17$ \\
\hline \multirow[t]{3}{*}{ Arrecife $\cdot 25 \mathrm{~m}$} & 2013 & $2 / 3$ & $4 / 5$ & $2 / 2$ & $3 / 3$ & $11 / 13$ \\
\hline & 2014 & $1 / 2$ & $2 / 3$ & $2 / 2$ & $1 / 7$ & $6 / 14$ \\
\hline & 2015 & $1 / 9$ & $1 / 3$ & $0 / 3$ & $3 / 4$ & $5 / 19$ \\
\hline \multicolumn{7}{|c|}{ Competidores (C. fulva, C. cruentata, E. adscensionis y E. guttatus) } \\
\hline & & JG & Real & Siju & Coco & Total \\
\hline \multirow[t]{3}{*}{ Reef $\bullet 15 \mathrm{~m}$} & 2013 & $0 / 5$ & $5 / 10$ & $0 / 2$ & $7 / 7$ & $12 / 24$ \\
\hline & 2014 & $7 / 7$ & $5 / 5$ & $5 / 6$ & $4 / 4$ & $21 / 22$ \\
\hline & 2015 & $5 / 8$ & $7 / 9$ & $7 / 9$ & $2 / 5$ & $21 / 19$ \\
\hline \multirow[t]{3}{*}{ Reef $\bullet 25 \mathrm{~m}$} & 2013 & $0 / 0$ & $3 / 3$ & $3 / 3$ & $2 / 5$ & $8 / 11$ \\
\hline & 2014 & $10 / 11$ & $3 / 12$ & $6 / 6$ & $7 / 12$ & $26 / 41$ \\
\hline & 2015 & $5 / 9$ & $5 / 8$ & $4 / 17$ & $10 / 15$ & $24 / 49$ \\
\hline \multicolumn{7}{|c|}{ Depredadores (M. bonaci, M. tigris y E. striatus) } \\
\hline \multirow{3}{*}{ Reef $\bullet 15 \mathrm{~m}$} & & JG & Real & Siju & Coco & Total \\
\hline & 2013 & $0 / 4$ & $1 / 5$ & $1 / 6$ & $1 / 3$ & $3 / 18$ \\
\hline & 2014 & $1 / 1$ & $2 / 6$ & $0 / 1$ & $1 / 5$ & $4 / 13$ \\
\hline & 2015 & $0 / 0$ & $0 / 0$ & $0 / 0$ & $0 / 4$ & $0 / 4$ \\
\hline \multirow[t]{3}{*}{ Reef $\bullet 25 \mathrm{~m}$} & 2013 & $1 / 1$ & $5 / 10$ & $2 / 2$ & $0 / 0$ & $8 / 13$ \\
\hline & 2014 & $1 / 2$ & $2 / 2$ & $3 / 3$ & $1 / 1$ & $8 / 8$ \\
\hline & 2015 & $0 / 3$ & $2 / 6$ & $1 / 2$ & $0 / 4$ & $3 / 15$ \\
\hline
\end{tabular}

Número de individuos contados y medidos en transectos/total de individuos medidos en la estación de muestreo. ND: No determinado. 

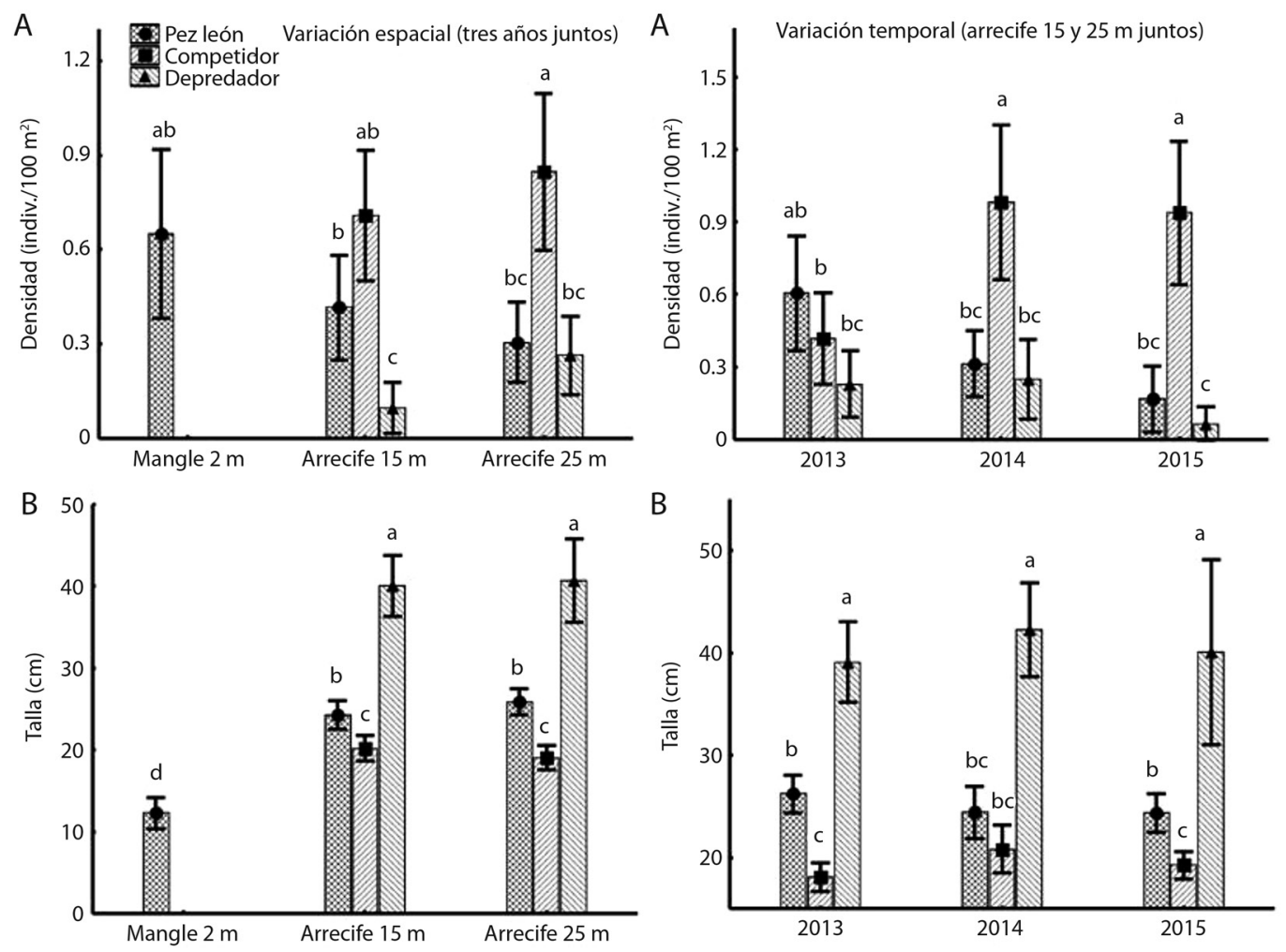

Fig. 2. Variación espacial (en la izquierda) y temporal (en la derecha) de la densidad promedio (A) y la talla promedio (B) del pez león en relación con la incidencia de potenciales competidores (C. fulva, C. cruentata, E. adscensionis y E. guttatus) y depredadores (M. bonaci, M. tigris y E. striatus) en del PNCSF. Las barras indican Media e intervalo de confianza (95\%) y las letras sobre las barras indican grupos estadísticamente homogéneos.

Fig. 2. Spatial (on the left) and temporal (on the right) variation of average density (A) and average size (B) of lionfish in relation to potential competitors (C. fulva, C. cruentata, E. adscensionis and E. guttatus) and predators (M. bonaci, M. tigris and E. striatus) incidence in of the NPCSF. Plot of Mean and Conf. Intervals (95\%) and letters on the top of the bar indicate statistically homogenous groups.

(Cuadro 1). Su densidad promedio por estación fue entre 0.4 y 1.2 indiv. $/ 100 \mathrm{~m}^{2}$ y su talla entre 16.3 y $23.4 \mathrm{~cm}$. La densidad promedio de competidores en arrecifes a $15 \mathrm{~m}$ fue de $0.7,0-3$ indiv. $/ 100 \mathrm{~m}^{2}$ con talla promedio de $20.2,10-40 \mathrm{~cm}$; y a $25 \mathrm{~m}$ la densidad promedio fue de $0.8,0-4$ indiv. $/ 100 \mathrm{~m}^{2}$ con talla de 19.0 , 10-45 cm (Fig. 2A y Fig. 2B). La densidad promedio de especies competidoras incrementó temporalmente $\left(\mathrm{F}_{(2,138)}=5.6 \mathrm{P}=0.03\right)$ y la talla promedio fue similar entre años y hábitats.

Se contaron 25 posibles meros depredadores de pez león y se midieron 71 individuos, las especies observadas fueron E. striatus, Mycteroperca tigris y Mycteroperca bonaci (Cuadro 1). Su densidad promedio por estación osciló entre $0.05-0.5$ indiv. $/ 100 \mathrm{~m}^{2}$ y su talla entre 35.8 y $43.1 \mathrm{~cm}$. La densidad promedio de especies depredadoras a $15 \mathrm{~m}$ fue de $0.1,0-2$ indiv. $/ 100 \mathrm{~m}^{2}$ y la talla de $40.1,17-57 \mathrm{~cm}$; y a $25 \mathrm{~m}$ la densidad fue de $0.26,0-2$ indiv./100 $\mathrm{m}^{2}$ con talla de 40.7, 23-90 cm (Fig. 2A y Fig. 2B). La densidad de depredadores fue menor en arrecifes a $15 \mathrm{~m}$ de profundidad $\left(\mathrm{F}_{(2,138)}=\right.$ $5.22 \mathrm{P}=0.01)$ y su talla no varió entre hábitats ni entre años. No obstante, en arrecife a $15 \mathrm{~m}$ la talla promedio de depredadores fue menor en el $2015\left(\mathrm{~F}_{(2,32)}=102.6 \mathrm{P}=0.03\right)$.

Incidencia del pez león en relación con la de meros competidores y depredadores: El análisis por hábitat mostró que existieron 
diferencias significativas $\left(\mathrm{F}_{(6,525)}=7.12 \mathrm{P}=\right.$ 0.00 ) en la densidad promedio del pez león con relación a la de meros, pero el test SNK no permitió definir qué medias fueron diferentes. No obstante, en arrecifes a 15 y $25 \mathrm{~m}$ los competidores presentaron valores promedios de densidad relativamente más altos, y en arrecife a $15 \mathrm{~m}$, la densidad promedio del pez león fue mayor que la de depredadores. La comparación de la densidad promedio entre años $\left(\mathrm{F}_{(8,423)}=10.82 \mathrm{P}=0.00\right)$ mostró que el pez león tuvo densidad similar a la de competidores y depredadores en el 2013, mientras que en el 2014 y 2015, la densidad de competidores fue superior a la del pez león y a la de los meros depredadores (Fig. 2A). El análisis espacial de la variación en la talla $\left(\mathrm{F}_{(6,390)}=71.4 \mathrm{P}=0.00\right)$ reflejó que el pez león en arrecifes a 15 y 25 m tuvo talla promedio mayor que la de los competidores, y menor que la de los depredadores. $\mathrm{El}$ análisis temporal de las tallas $\left(\mathrm{F}_{(8,348)}=39.41\right.$ $\mathrm{P}=0.00)$ reflejó que durante los tres años de muestreo, el pez león tuvo talla inferior a la de depredadores y mayor o similar a la de los competidores (Fig. 2B).

\section{DISCUSIÓN}

El pez león se observó por primera vez en arrecifes de los Cayos de San Felipe en agosto 2009 y después de cuatro o cinco años de su arribo, los valores medios encontrados de su densidad y su talla, fueron similares a los registrados en otros arrecifes de Cuba (Chevalier, et al., 2014; García-Rodríguez et. al, 2015) y del Gran Caribe (Whitfield et al., 2007; Green \& Côté, 2009; Valdivia et al., 2014; Sabido-Itzá1, Medina-Quej, Jesús-Navarrete, Gómez-Poot, \& García-Rivas, 2016). No obstante, los valores de la densidad del pez león en el PNCSF fueron relativamente bajos si se comparan con los registrados por esos mismos autores. Para otras especies de peces (no pez león) se ha observado que limitaciones de alimento o hábitat pueden llevar a que la población exceda su capacidad de carga, provocando el descenso de la abundancia, como parte de la dinámica natural del proceso de colonización (Mumby et al., 2011). Sin embargo, dada la baja abundancia del pez león encontrada en el PNCSF y la aparente disponibilidad de refugios y alimento en sus arrecifes, es poco probable que el fenómeno antes descrito haya tenido lugar en el área de estudio.

La incidencia de especies de meros nativos potencialmente competidoras (Whitfield et al., 2007) y depredadoras (Mumby et al., 2011) es otro factor que se ha considerado como posible regulador del éxito de la colonización del pez león en los arrecifes (Albins, 2013; Bejarano et al., 2015). En el PNCSF la densidad de las especies competidoras fue igual o superior que la del pez león, lo cual se corresponde con la relación encontrada en otros arrecifes de la costa norte y sur de Cuba (Chevalier et al., 2014), y en la costa este de Estados Unidos (Whitfield et al., 2007). Esta equidad puede ser un indicador de que hasta el momento en el PNCSF el pez león no ha superado en competencia a estas especies nativas. No obstante, las tallas del pez león en el PNCSF fueron ligeramente superiores a las de los meros nativos, por lo que existe la posibilidad de que a largo plazo se establezcan relaciones de competencia por alimento más desventajosas para las especies nativas.

Dentro del PNCSF, probablemente debido al uso pesquero que ha tenido la zona por más de 50 años (Claro et al., 2001), la densidad y la talla de meros nativos depredadores fue muy baja y se mantuvo por debajo de la encontrada en zonas protegidas de la pesca en Cuba y el Caribe (Mumby et al., 2011; Pina-Amargós, González-Sansón, Martín-Blanco, \& Valdivia, 2014; Valdivia et al., 2014). En áreas marinas donde la densidad de los meros es alta, se ha podido probar el efecto regulador de los meros depredadores sobre la abundancia del pez león (Whitfield et al., 2007; Mumby et al., 2011). Sin embargo, en el PNCSF los resultados del estudio no ofrecieron información para considerar que este mecanismo de control esté actuando. Motivo por el que se recomienda continuar el seguimiento de la densidad de meros potenciales depredadores y realizar estudios de su contenido estomacal 
para conocer si realmente están consumiendo pez león en el área.

En los manglares del PNCSF se encontró alta estabilidad en la densidad del pez león y dominancia de ejemplares con tallas consideradas por Gardner, Frazer, Jacoby and Yanong (2015) como juveniles. Esto permite sugerir que estos manglares fungen como zonas de cría $\mathrm{y}$, posiblemente, como fuente permanente para el reclutamiento del pez león en los arrecifes del parque. Por tanto, se considera de alta prioridad continuar el monitoreo en los manglares y comenzar el estudio del contenido estomacal del pez león en este hábitat con vistas a conocer si la voracidad y la composición de su dieta (Morris \& Akins, 2009; Barbour, Montgomery, Adamson, Diaz-Ferguson, \& Silliman, 2010; Albins, 2013) representa una amenaza para la langosta espinosa o peces que constituyen recursos pesqueros importantes de la zona suroccidental de Cuba y utilizan los manglares en etapas juveniles (Claro et al., 2001; Cruz, Díaz, Báez, \& Adriano, 2001). Otra estrategia de manejo puede ser comenzar la remoción del pez león en los manglares del PNCSF, con vistas a disminuir su reclutamiento en los arrecifes y con ello, controlar aún más su impacto en el arrecife y sobre las especies de importancia comercial para el sector pesquero.

Estudios realizados en el Caribe apoyan este tipo de acciones, pues han concluido que la extracción sistemática del pez león pueden llevar al control de sus poblaciones (Barbour, Allen, Frazer, \& Sherman, 2011; Frazer, Jacoby, Edwards, Barry, \& Manfrino, 2012), tanto si se extraen adultos (Bejarano et al., 2015) como juveniles (Morris, Shertzer, \& Rice, 2011). No obstante, la densidad relativamente baja del pez león encontrada en el PNCSF y su tendencia temporal a descender en ausencia de acciones extractivas (para lo cual este trabajo no ofrece explicación), sugieren que otros factores locales pueden estar incidiendo en la regulación de la abundancia del pez león. Una posible explicación puede asociarse al criterio de que los pargos mayores de $20 \mathrm{~cm}$ y las langostas adultas, pueden considerarse potenciales depredadores o competidores por el espacio, respectivamente (Green, 2013). El PNCSF está ubicado en la zona de pesca de langosta más productiva de Cuba (Cruz et al., 2001) y en sus inmediaciones se ubica un sito de desove de pargos que provoca alta concentración de Lutjanus analis y L. cyanoptera durante la época reproductiva (Claro \& Lindeman, 2003), por lo que pargos y langostas se pueden considerar muy abundantes en el área y es poco probable que no interactúen con el pez león. Por tanto, la idea es potencialmente posible y puede quedar abierta como interrogante para futuros estudios en el parque.

\section{AGRADECIMIENTOS}

Se agradece al CINVESTAV y al Consejo Nacional de Ciencia y Tecnología (CONACyT) de México por el financiamiento recibido y la beca otorgada para el Doctorado de Elena de la Guardia (N. ${ }^{\circ}$ de Beca 307812). Agradecemos a los trabajadores del PNCSF y el apoyo financiero y en equipamiento recibido del Fondo Global para Medioambiente, de la operación WALLACEA en Cuba, de la Empresa para la Protección de la Flora y la Fauna, del Centro Nacional de Áreas Protegidas de Cuba y del Centro de Investigaciones Marinas de la Universidad de La Habana.

\section{RESUMEN}

El primer avistamiento del pez león en el Parque Nacional "Cayos de San Felipe" en el 2009 podría amenazar la diversidad de sus ecosistemas marinos y la capacidad de brindar servicios. Para analizar la incidencia de la invasión del pez león en el área, se realizó un muestreo anual entre 2013 y 2015. La abundancia y la talla del pez león se estudió en manglares a través de censos visuales en diez transectos de $30 \times 2 \mathrm{~m} /$ estación y en arrecifes (15 y $25 \mathrm{~m}$ de profundidad) con estéreo video en seis transectos de 50x2 $\mathrm{m} /$ estación. En arrecifes también se estimó incidencia de posibles competidores y depredadores nativos. En los tres años de estudio, la densidad promedio del pez león varió entre $0.0-1.3$ indiv. $/ 100 \mathrm{~m}^{2}$ por estación de muestreo y no fue significativamente diferente entre hábitats (mangle - 0.6 indiv. $/ 100 \mathrm{~m}^{2}$, arrecifes a $15 \mathrm{~m}-0.4$ indiv. $/ 100 \mathrm{~m}^{2}$ y arrecifes a $25 \mathrm{~m}-0.3$ indiv. $/ 100 \mathrm{~m}^{2}$ ). La densidad de pez león fue igual o menor que la de competidores e igual o mayor que la de depredadores en ambas profundidades. La densidad del pez león en manglares y arrecifes a $25 \mathrm{~m}$ se mantuvo 
temporalmente estable, mientras que en arrecifes a $15 \mathrm{~m}$ disminuyó. Se observó que el incremento temporal de la abundancia de competidores y la densidad de depredadores no cambió durante el monitoreo. La talla del pez león varió entre 5 y $39 \mathrm{~cm}$, siendo su promedio en manglares $(12.6 \mathrm{~cm})$ menor que en arrecifes $(25.2 \mathrm{~cm})$ y no mostró variaciones entre años. La talla de pez león en arrecifes fue más alta que la de competidores y menor que la de depredadores. Los resultados mostraron que en el parque: 1) los manglares son zonas de cría del pez león, 2) la incidencia del pez león en arrecifes no es tan alta como en otras áreas de Cuba y el Caribe y 3) la abundancia del pez león en arrecifes tendió a disminuir en el tiempo sin intervención de acciones extractivas o presencia de abundancia alta de meros nativos de tallas grandes. Considerando los resultados, se hacen recomendaciones para continuar el monitoreo e investigar los efectos del pez león y los factores que estan regulando su incidencia en el parque.

Palabras clave: pez león, especie invasora, arrecifes de coral, Caribe, talla, densidad.

\section{REFERENCIAS}

Arias-González, J. E., González-Gandara, C., Cabrera, J. L., \& Christensen, V. (2011). Predicted impact of the invasive lionfish Pterois volitans on the food web of a Caribbean coral reef. Environmental Research, $111,917-925$.

Albins, M. A. (2013). Effects of invasive Pacific red lionfish Pterois volitans versus a native predator on Bahamian coral-reef fish communities. Biological Invasions, 15, 29-43.

Barbour, A. B., Montgomery, M. L., Adamson, A. A., DiazFerguson, E., \& Silliman, B. R. (2010). Mangrove use by the invasive lionfish Pterois volitans. Marine Ecology Progress Series, 40, 291-294.

Barbour, A. B., Allen, M. S., Frazer, T. K., \& Sherman, K. D. (2011). Evaluating the potential efficacy of invasive lionfish (Pterois volitans) removals. PLOS ONE, 6, 1-7.

Bejarano, S., Lohr, K., Hamilton, S., \& Manfrino, C. (2015). Relationships of invasive lionfish with topographic complexity, groupers, and native prey fishes in Little Cayman. Marine Biology, 162, 253-266.

Betancur, R., Hines, A., Acero, G. A., Orti, P., Wilbur, A. E., \& Freshwater, D. W. (2011). Reconstructing the lionfish invasion: insights into Greater Caribbean biogeography. Journal of Biogeography, 38, 1281-1293.

Chevalier, P., Cabrera, E., Caballero, H., Corrada, R., Fernández, A., Cobián, D., \& García, A. (2014). Distribución, abundancia y relaciones ecológicas del pez león (Pterois volitans/miles: Scorpaenidae) en Cuba. Gulf and Caribbean Fisheries Institute, 66, 178-179.
Claro, R. \& Lindeman, K. C. (2003). Spawning aggregation sites of snapper and grouper species (Lutjanidae and Serranidae) on the insular shelf of Cuba. Gulf and Caribbean Research Supplement, 57, 1-15.

Claro, R., Baisre, J. A., Lindeman, K. C., \& García-Arteaga, J. P. (2001). Cuban fisheries: Historical trends and current status. In R. Claro, K. C. Lindeman, \& L. R. Parenti (Eds.), Ecology of Marine Fishes of Cuba (pp. 194-219). Washington, D. C.: Smithsonian Institution Press.

Cruz, R., Díaz, E., Báez, M., \& Adriano, R. (2001). Variability in recruitment of multiple life stages of the Caribbean spiny lobster, Panulirus argus, in the Gulf of Batabanó, Cuba. Marine and Freshwater Research, 52, 1263-1270.

Côté, I. M., \& Green, S. J. (2012). Potential effects of climate change on a marine invasion: The importance of current context. Current Zoology, 58, 1-8.

Frazer, T. K., Jacoby, C. A., Edwards, M. A., Barry, S. C., \& Manfrino, C. M. (2012). Coping with the Lionfish Invasion: Can Targeted Removals Yield Beneficial Effects? Reviews in Fisheries Science, 20, 185-191.

García-Rodríguez, A., Chevalier, P., Cabrera E., Caballero H., \& Hernández, J. (2015). Densidad y biomasa de Pterois volitans/miles (Teleostei: Scorpaenidae) en arrecifes del litoral oeste de la Habana, Cuba. Revista de Investigaciones Marinas, 35, 21-36.

Gardner, P. G., Frazer, T. K., Jacoby, C. A., \& Yanong, R. P. (2015). Reproductive biology of invasive lionfish (Pterois spp.). Frontiers in Marine Science, 2, 1-10.

Green, S. J. (2013). El monitoreo: una actividad fundamental. En J. A. Morris Jr. (Ed.), El pez león invasor : guía para su control y manejo (pp.59-982). Marathon, Florida, USA: Gulf and Caribbean Fisheries Institute Special Publication Series 2.

Green, S. J., \& Cote, I. M. (2009). Record densities of Indo-Pacific lionfish on Bahamian coral reefs. Coral Reefs, 28, 107-107.

Harvey, E., Fletcher, D., Shortis, M. R., \& Kendrick, G. A. (2004). A comparison of underwater visual distance estimates made by SCUBA divers and a stereo-video system: implications for underwater visual census of reef fish abundance. Marine and Freshwater Research, 55, 573-580.

Morris, Jr., \& Akins, L. (2009). Feeding ecology of invasive lionfish (Pterois volitans) in the Bahamian archipelago. Environmental Biology of Fishes, 86, 389-398.

Morris, J. A., Shertzer, K. W., \& Rice, J. A. (2011). A stage-based matrix population model of invasive lionfish with implications for control. Invasion Note, Biological Invasions, 13, 7-12. 
Mumby, P. J., Harborne, A. R., \& Brumbaugh, D. R. (2011). Grouper as a natural biocontrol of invasive lionfish. PLOS ONE, 6:e21510.

Pina-Amargós, F., González-Sansón, G., Martín-Blanco, F., \& Valdivia, A. (2014). Evidence for protection of targeted reef fish on the largest marine reserve in the Caribbean. PeerJ 2:e274; DOI 10.7717

Underwood, A. J. (1997). Experiments in ecology. Their logical design and interpretation using analysis of variance. Cambridge: Cambridge University Press.

Sabido-Itzá1, M. M., Medina-Quej, A., Jesús-Navarrete, A., Gómez-Poot, J. M., \& García-Rivas, M. C. (2016). La estructura de tallas como evidencia del establecimiento de Pterois volitans (Scorpaeniformes: Scorpaenidae) en el sur del Caribe mexicano. Revista de Biología Tropical, 64, 353-362.
SeaGIS. (2011). Retrieved 2011-06-27 from www.seagis. com.au

Valdez-Moreno, M., Quintal-Lizama, C., Gómez-Lozano, R., \& García-Rivas, M. C. (2012). Monitoring an alien invasion: DNA barcoding and the identification of lionfish and their prey on coral reefs of the Mexican Caribbean. PloS One, 7, 1-8.

Valdivia, A., Bruno, J. F., Cox, E. C., Hackerott, S., \& Green, S. J. (2014). Re-examining the relationship between invasive lionfish and native grouper in the Caribbean. PeerJ 2:e348; DOI 10.7717

Whitfield, E. P., Hare, A. J., Andrew, W. D., Harter, L. S., Muñoz, C. R., \& Addisson, M. C. (2007). Abundance estimates of the Indo-Pacific lionfish Pterois volitans/ miles complex in the Western North Atlantic. Biological Invasions, 9, 53-64. 
